\title{
Unraveling the Mechanism of the Covalent Coupling Between Terminal Alkynes on a Noble Metal
}

Jonas Björk, Yi-Qi Zhang, Florian Klappenberger, Johannes V. Barth and Sven Stafström

\section{Linköping University Post Print}

\section{Tweet}

N.B.: When citing this work, cite the original article.

Original Publication:

Jonas Björk, Yi-Qi Zhang, Florian Klappenberger, Johannes V. Barth and Sven Stafström, Unraveling the Mechanism of the Covalent Coupling Between Terminal Alkynes on a Noble Metal, 2014, The Journal of Physical Chemistry C, (118), 6, 3181-3187.

http://dx.doi.org/10.1021/jp5002918

Copyright: American Chemical Society http://pubs.acs.org/

Postprint available at: Linköping University Electronic Press http://urn.kb.se/resolve?urn=urn:nbn:se:liu:diva-104257 


\section{Unraveling the Mechanism of the Covalent}

\section{Coupling Between Terminal Alkynes on a Noble}

\section{Metal}

Jonas Björk, ${ }^{* \dagger}$ Yi-Qi Zhang, ${ }^{\ddagger}$ Florian Klappenberger, ${ }^{\ddagger}$ Johannes V. Barth, ${ }^{\ddagger}$ and Sven Stafström ${ }^{\dagger}$

Department of Physics, Chemistry and Biology, IFM, Linköping University, 58183

Linköping, Sweden, and Physik Department E20, Technische Universität München, 85748

Garching, Germany

E-mail: jonas.bjork@liu.se

${ }^{*}$ To whom correspondence should be addressed

$\dagger$ Department of Physics, Chemistry and Biology, IFM, Linköping University, 58183 Linköping, Sweden

${ }^{\ddagger}$ Physik Department E20, Technische Universität München, 85748 Garching, Germany 


\begin{abstract}
The mechanism of the newly reported route for surface-assisted covalent coupling of terminal alkynes on $\mathrm{Ag}(111)$ is unraveled by density functional theory based transition state calculations. We illustrate that the reaction path is fundamentally different from the classical coupling schemes in wet chemistry. It is initiated by the covalent coupling between two molecules instead of single-molecule dehydrogenation. The silver substrate is found to play an important role stabilizing the intermediate species by chemical bonds, although it is hardly active electronically in the actual coupling step. The dimer intermediate is concluded to undergo two subsequent dehydrogenation processes expected to be rate-limiting according to the comparatively large barriers, which origin is discussed.
\end{abstract}

\title{
Keywords
}

covalent assembly, surface chemistry, reaction mechanisms, DFT calculations, transition state theory 


\section{Introduction}

Assembling organic molecules into covalent nanoarchitectures is becoming a very active research area in surface and nanoscale science. ${ }^{1-3}$ The potential of this extremely versatile approach has been demonstrated in the fabrication of one and two-dimensional hydrocarbon materials with atomic precision ${ }^{4-10}$ and was listed in the graphene roadmap as a promising strategy toward obtaining high-quality nanographenes. ${ }^{11}$ In principle, custom-made materials can be obtained, depending on the molecular building blocks, the coverage of molecules, the temperature, and the nature of the underlying substrate. The major goal is the controlled design of polymers extended in one and two dimensions by the molecular building blocks. ${ }^{1,12}$ However, to reach this milestone, a thorough theoretical understanding of the involved on-surface reactions is crucial.

Up to now, the main achievements of covalent assembly on surfaces have been based on using halogen-substituted molecular building blocks. ${ }^{13}$ The approach exploits that the halogen atoms are more easily abstracted than their hydrogen counterparts. This results in species featuring unsaturated carbon atoms that establish a covalent bond after an intermediate diffusion process. ${ }^{14}$ However, the necessary diffusion of both the chemisorbed intermediates and the halogen byproducts ${ }^{15,16}$ can obstruct the creation of the targeted hydrocarbon-pure material.

Recently, we introduced homo-coupling of terminal alkynes on a nobel metal surface as a new tool for covalent construction of nanoarchitectures. ${ }^{17}$ The generality of this approach was demonstrated by its application to different organic building-blocks ${ }^{17-19}$ and on the differently reactive surfaces $\mathrm{Ag}(111),{ }^{17,18} \mathrm{Au}(111)^{18}$ and $\mathrm{Cu}(111) .{ }^{18,19}$ The reaction takes place under mild and clean conditions, with hydrogen as only byproduct released from the

surface. However, several unwanted side-reactions have been reported, ${ }^{18,20}$ reducing the product yield. Thus, for exploiting the full potential of this construction tool, a systematic theoretical investigation is necessary.

Furthermore, previous investigations of other interfacial covalent assembly schemes ${ }^{14,21-24}$ 


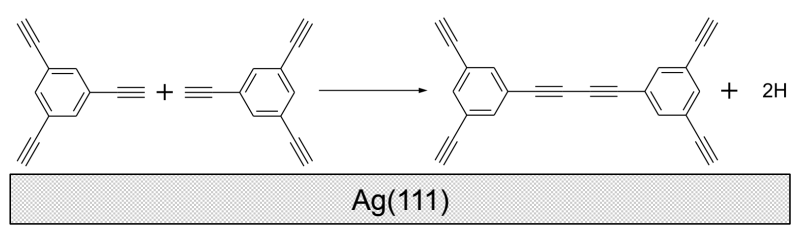

Scheme 1: The coupling reaction of two 1,3,5-triethynyl-benzene (TEB) molecules into a TEB dimer, which takes place on the $\mathrm{Ag}(111)$ surface.

revealed that chemical intuition often fails to predict how a reaction proceeds under onsurface conditions, because pathways not accessible in conventional wet chemistry are often operative. Therefore, theoretical approaches providing detailed insights by examining in depth the underlying mechanisms, are of the utmost importance. ${ }^{25}$

The reaction discussed here is formally reminiscent of the classic Glaser-Hay coupling scheme, ${ }^{26,27}$ but can also be interpreted as a basic step of surface-confined acetylide chemistry. ${ }^{28-30}$ In solution, the reaction is well-known to proceed by dehydrogenating the terminal alkyne with a $\mathrm{Cu}$ ion and establishing a $\mathrm{Cu}$-acetylide intermediate. ${ }^{31}$ In the presence of $\mathrm{Ag}$ ions, the related Ag-acetylides can be formed ${ }^{28}$ however indication of the Ag-related synthesis of the butadiyne moiety has not been reported so far. At the same time, from the perspective of surface science, it is an established fact that the dissociative adsorption of hydrogen on $\mathrm{Ag}(111)$ is endothermic, ${ }^{32}$ and atomic hydrogen desorbs recombinatively at temperatures below $200 \mathrm{~K} \cdot{ }^{33}$ How the reaction will proceed at a $\mathrm{Ag}(111)$ surface is therefore a highly intricate question. In this respect, first hints regarding the coupling pathway have been uncovered, ${ }^{17,34}$ but the complete reaction mechanism remains elusive.

Here, we present a detailed analysis of the covalent coupling reaction using density functional theory based transition state calculations. We have focused on the dimerization of two 1,3,5-triethynyl-benzene (TEB) monomers, illustrated in Scheme 1. By scrutinizing possible reaction pathways, the most favorable mechanism is identified, which is fundamentally different from the classical Glaser-Hay coupling in wet chemistry. ${ }^{31,35-37}$ 


\section{Methods}

\section{Computational details}

Our theoretical toolbox used to approach the problem is based on periodic density functional theory (DFT) using the VASP code. ${ }^{38-40}$ The van der Waals density functional (vdW-DF) ${ }^{41}$ was used, as nonlocal correlations are required to describe the forces responsible for Ag-C interactions, while the optimized form of the Becke 86 functional $^{42}$ was used to describe local correlation and semi-local exchange. The $\mathrm{Ag}(111)$ surface has been represented in a $p(10 \times 9)$ supercell, containing four atomic layers separated by a $15 \AA$ vacuum. The bottom two Ag layers were kept fixed, while the remaining atoms were allowed to relax. Structural optimizations were performed until the forces acting on all atoms were smaller than $0.01 \mathrm{eV} / \AA$.

Transition states were calculated in a two-step procedure. First, the Climbing Image Nudged-Elastic Band (CI-NEB) method $^{43,44}$ was used to get an initial guess of the transition state. The transition path was optimized until the forces acting perpendicular to the path were converged typically to below $0.1-0.2 \mathrm{eV} / \AA$. The rough estimate of the transition path from the CI-NEB calculations were used as input for the Dimer method. ${ }^{45,46}$ The initial guess of the central image (i.e. the transition state) was taken as the highest energy point on the CINEB path. The dimer was obtained by calculating the vector going between the two images adjacent to the highest energy point in the CI-NEB path, and by adding/subtracting this vector to/from the central image. The structural optimization of the dimer was performed until the forces acting on the atoms on the central image were smaller than $0.02 \mathrm{eV} / \AA$. In

the case of the $\mathbf{T S}_{\mathbf{d e H} \mathbf{2}}$, the forces were converged to below $0.01 \mathrm{eV} / \AA$ to ensure accurate vibrational frequencies entering the quasiharmonic approximation.

The scanning tunneling microscopy (STM) images were simulated using the TersoffHamann approximation ${ }^{47}$ with the implementation by Lorente and Persson. ${ }^{48}$ 


\section{STM experiment}

The topographic data of the TEB dimer on $\mathrm{Ag}(111)$ were acquired at $5.5 \mathrm{~K}$ using a homemade Besocke-type STM working under ultra-high vacuum (UHV) condition with a base pressure better than $2 \times 10^{-10}$ mbar. The samples used for STM measurement were prepared in situ by depositing TEB molecules onto a $\mathrm{Ag}(111)$ surface held at $\sim 330 \mathrm{~K}$, precleaned by $\mathrm{Ar}^{+}$sputtering and annealing, using organic molecular beam epitaxy with a high molecular flux (partial pressure $\sim 10^{-8}$ mbar). ${ }^{17}$

\section{Results and discussion}

For the TEB dimerization, the first question to answer is whether the TEB units first dissociate and concomitantly couple on the surface, or if two TEB units couple first, followed by the abstraction of hydrogen atoms from the dimeric product at a later stage. Beginning with the direct dissociation, on the atomically flat $\operatorname{Ag}(111)$ surface, the dehydrogenation is associated with a barrier as large as $1.82 \mathrm{eV}$, Figure 1a. One may also consider that the reaction is catalyzed by Ag adatoms, Figure 1b. The reference energy of the adatom was taken as an isolated atom adsorbed on the surface. Using this reference for the adatoms, the initial state (IS) of this path is only slightly more stable than the IS of the path associated with the dissociation on the flat surface. In other words, there is no significant attraction between the adatoms and TEB molecules, and the dehydrogenation barrier exceeds the one

on the atomically flat surface. Furthermore, this reaction is dependent on the availability of Ag adatoms, making it significantly less likely than the dehydrogenation on the flat surface. As an alternative to these dissociation processes, we considered the possibility that the coupling reaction is initiated by a tautomerization step, in which the terminal C-atom becomes unsaturated by moving its H-atom to the adjacent C-atom (Figure S1). The tautomerization has a barrier of $1.93 \mathrm{eV}$, close to that of the adatom-assisted hydrogen abstraction.

In addition, we considered the possibility that an already removed $\mathrm{H}$ atom catalyzes the 
dehydrogenation reaction (Figure 1c), by forming an $\mathrm{H}_{2}$ molecule following the dissociation. Interestingly, this has a positive impact on the reaction, as it lowers the barrier to $1.39 \mathrm{eV}$. However, as will be illustrated below, a majority of the TEB monomers will follow a different reaction path.

Instead of first splitting off hydrogen, we find that the reaction is much more likely to be initiated by the coupling between two TEB molecules. Figure 2 illustrates the covalent bond
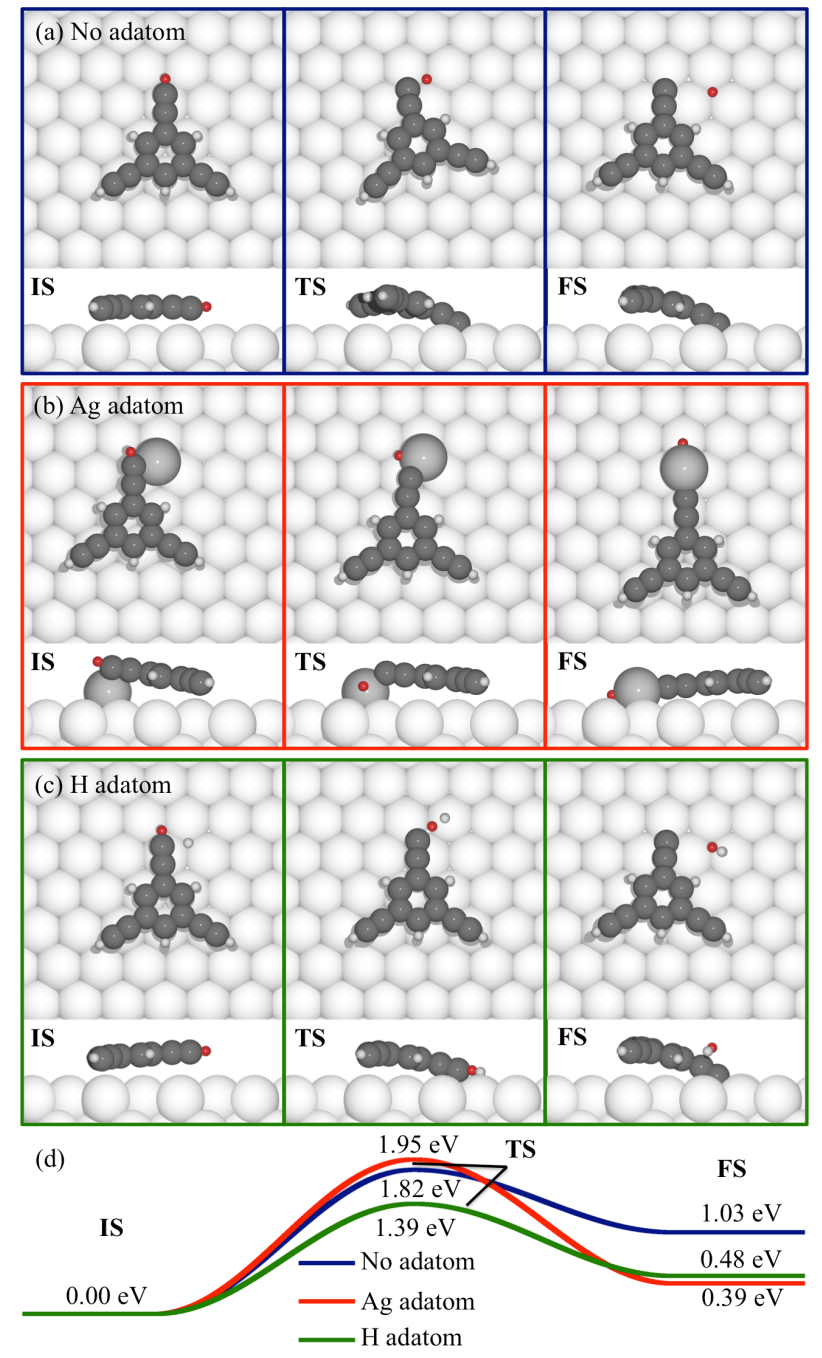

Figure 1: Potential pathways for the dehydrogenation of a terminal alkyne of a TEB molecule on $\mathrm{Ag}(111)$, depicting top and side views of initial state (IS), transition state (TS), and final state (FS) for splitting-off a hydrogen atom (a) on the atomically flat surface, (b) in the presence of an Ag adatom, and (c) in the presence of a hydrogen adatom. The abstracted hydrogen atom is rendered in red to ease comparison. The energy profiles for the reactions are shown in $(d)$. 


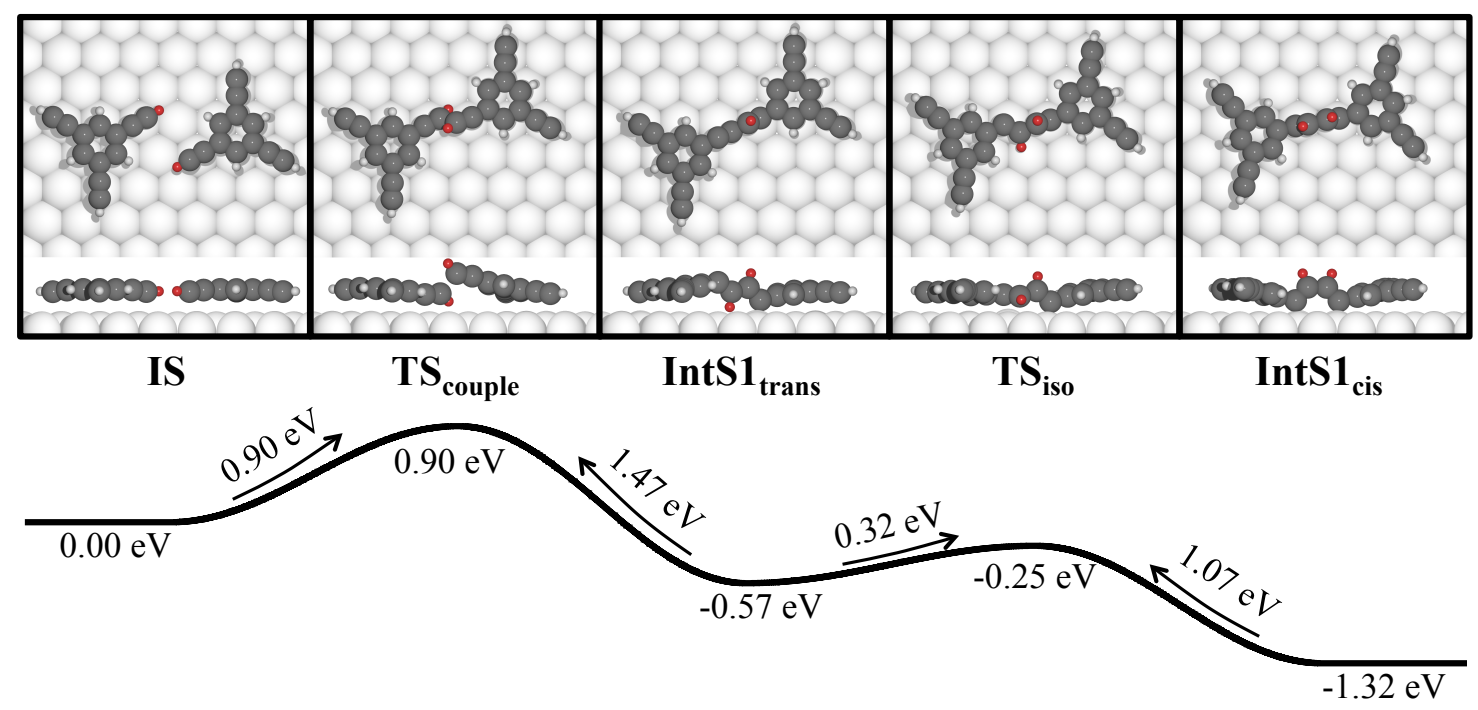

Figure 2: Reaction path for the direct coupling of two TEB molecules on $\operatorname{Ag}(111)$. The coupling transition state $\mathbf{T S} \mathbf{S}_{\text {couple }}$ leads to the two isomers $\mathbf{I n t S} \mathbf{1}_{\text {trans }}$ and $\mathbf{I n t S} \mathbf{1}_{\text {cis }}$, which are separated by an isomerization transition state $\mathbf{T S}_{\mathbf{i s o}}$. The hydrogen atoms of the two reacting alkynes are rendered in red to ease comparison. Energies of local minima and saddle points are given with respect to the initial state IS.

formation, as well as an isomerization process of the resulting compound. Strikingly, the barrier to merge two molecules is $0.90 \mathrm{eV}$, just half the barrier of the direct dehydrogenation of TEB on the atomically flat $\mathrm{Ag}(111)$ surface, and $0.5 \mathrm{eV}$ lower than the $\mathrm{H}$ adatom driven dissociation.

To study the impact of the calculated energy barriers on the reaction kinetics we estimated the reaction rates for the dehydrogenation of a TEB molecule, as well as for the direct recombination of two molecules. Except for the recombination, the rates were obtained from the Arrhenius equation $\nu=A \exp \left(-E_{\text {barrier }} / k_{\mathrm{B}} T\right)$, with the commonly used pre-factor $A=10^{13} \mathrm{~s}^{-1}$. It was recently shown ${ }^{14}$ that the rate of recombination $\nu_{\text {recomb }}$ can be expressed as a function of the rates of the coupling between two molecules ( $\left.\nu_{\text {couple }}\right)$ and molecular diffusion $\left(\nu_{\text {diffuse }}\right)$ as

$$
v_{\text {recomb }}=\theta \frac{\nu_{\text {couple }} \nu_{\text {diffuse }}}{\nu_{\text {couple }}+\nu_{\text {diffuse }}}
$$

where $\theta$ is the coverage of molecules, with $\theta=1$ corresponding to a fully covered surface. 


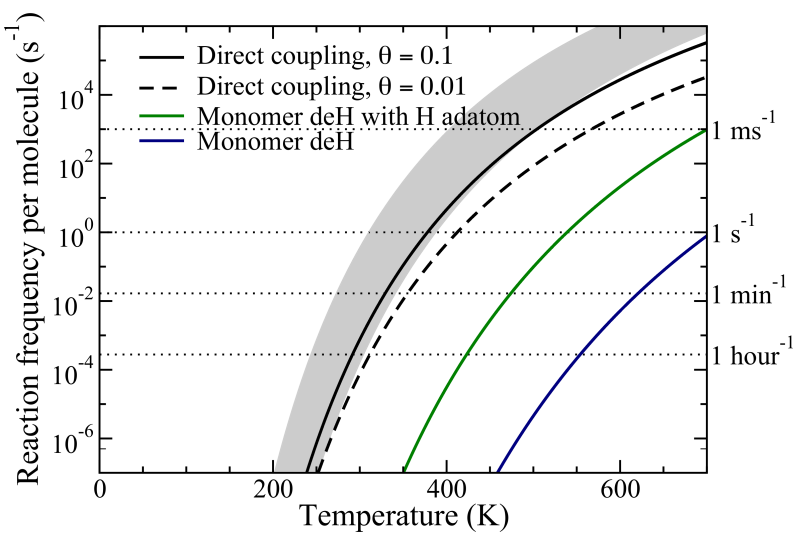

Figure 3: Reaction rates, obtained from the Arrhenius equation $\nu=A \exp \left(-E_{\text {barrier }} / k_{\mathrm{B}} T\right)$ using the calculated energy barriers as input. The recombination rates for the directly coupling of two TEB molecules were calculated with Eq. 1 for two different molecular coverages, using the coupling rates (calculated from an energy barrier of $0.90 \mathrm{eV}$, see Figure. 2) and diffusion rates (calculated from an energy barrier of $0.15 \mathrm{eV}$, see Supporting Information). The monomer dehydrogenation $(\mathrm{deH})$ rates were calculated both for the clean surface and in the presence of an $\mathrm{H}$ adatom, with barriers of $1.82 \mathrm{eV}$ and $1.39 \mathrm{eV}$, respectively (Figure 1). The shaded area shows the desorption rate, calculated from the experimentally estimated adsorption energy of TEB on $\mathrm{Ag}(111)$ of $0.8-1.0 \mathrm{eV} .{ }^{49}$

The diffusion barrier of TEB is $0.15 \mathrm{eV}$ (see supporting information), resulting in $\nu_{\text {diffuse }} \gg$

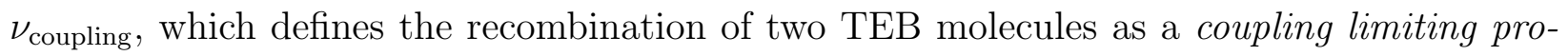
cess. $^{25}$ Note that in principle one would need to consider concentration and diffusion also for the dehydrogenation catalyzed by an $\mathrm{H}$ adatom, both for adatoms and molecules. To simplify the analysis we have expressed this reaction only in terms of the Arrhenius equation of the dehydrogenation barrier, which gives an upper limit of the rate for this reaction.

Figure 3 compares the resulting reaction rates for the direct coupling of two molecules, with the dehydrogenation on the atomically flat $\mathrm{Ag}(111)$ surface and the dehydrogenation catalyzed by an $\mathrm{H}$ adatom. The direct coupling reaction has reaction rates several orders of magnitudes larger than the dehydrogenation reactions at $330 \mathrm{~K}$. Thus, it is clear that the direct coupling is the most likely initial step of the overall reaction.

The initial coupling mechanism also has to be put into perspective with the experiment. From our earlier work ${ }^{17}$ it is known that the desorption of TEB monomers is competing with the coupling reaction. In fact, desorption prevails at $300 \mathrm{~K}$, while for triggering dimerization 
$\sim 330 \mathrm{~K}$ are required. It has been estimated that the experimental desorption energy of a TEB molecule from $\mathrm{Ag}(111)$ is in the range of $0.8-1.0 \mathrm{eV} .{ }^{49}$ The range of desorption rates obtained from the Arrhenius equation for these desorption energies is indicated by the shaded area in Figure 3. It is evident that the desorption and coupling occur at similar conditions, but the desorption is a slightly more likely process, in agreement with the experimental observations.

The covalent, non-dehydrogenated, dimer is considered as the first intermediate state in the overall reaction, and can exist both in a trans $\left(\mathbf{I n t} \mathbf{S} \mathbf{1}_{\text {trans }}\right)$ and a cis $\left(\mathbf{I n t} \mathbf{S} \mathbf{1}_{\text {cis }}\right)$ configuration. IntS1 $1_{\text {cis }}$ is the more stable of the two, due to its two $\mathrm{C}$-Ag bonds, while for IntS1 $1_{\text {trans }}$ only one carbon atom bonds to the surface. Thus, the transition state associated with the coupling of two TEB molecules $\left(\mathbf{T S}_{\text {couple }}\right)$ is likely leading to $\mathbf{I n t S} \mathbf{1}_{\text {cis }}$. This happens either via $\mathbf{I n t S} \mathbf{1}_{\text {trans }}$ and the isomerization transition state $\mathbf{T} \mathbf{S}_{\text {iso }}$, or directly without going through the trans isomer. Note that the inverse barrier separating $\mathbf{I n t S} \mathbf{1}_{\text {cis }}$ from the initial state $(\mathbf{I S})$ is $2.22 \mathrm{eV}$, and $\mathbf{I n t S} \mathbf{1}_{\text {cis }}$ is more stable than $\mathbf{I S}$ by $1.32 \mathrm{eV}$. At reaction equilibrium, IntS1 $\mathbf{1}_{\text {cis }}$ is $\sim 10^{20}$ times more probable than IS (calculated from the Boltzmann factor for $1.32 \mathrm{eV}$ at $330 \mathrm{~K}$ ). Therefore, once two TEB molecules have coupled, it is very unlikely that they will dissociate into separate units.

To investigate the role of the $\mathrm{Ag}(111)$ surface in the coupling of two TEB molecules, the electron density difference profile of the coupling transition state $\mathbf{T} \mathbf{S}_{\text {couple }}$ was considered. Two specific electron density differences were calculated as

$$
\Delta \rho_{\mathbf{a}}=\rho_{\text {full }}-\rho_{\text {slab }}-\rho_{\text {mol1 }+ \text { mol } 2},
$$

and as

$$
\Delta \rho_{\mathbf{b}}=\rho_{\text {full }}-\rho_{\text {slab }}-\rho_{\text {mol1 }}-\rho_{\text {mol } 2},
$$

where $\rho_{\text {full }}$ is the electron density of the full system, $\rho_{\text {slab }}$ the electron density of the Ag slab, $\rho_{\text {mol1+mol2 }}$ the electron density of the two interacting molecules (without slab), and $\rho_{\text {mol1 }}$ and 


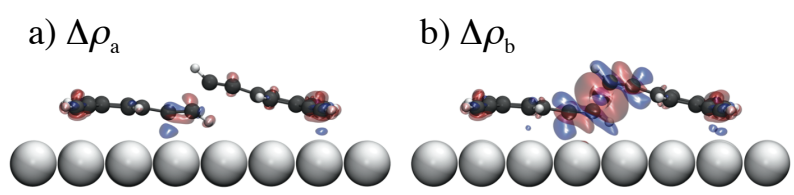

Figure 4: Electron density difference plots of the coupling transition state $\mathbf{T} \mathbf{S}_{\text {couple }}$, illustrating the redistribution of the electron density due to the coupling of two TEB molecules, induced by (a) molecule-surface interactions, and (b) both molecule-surface and moleculemolecule interactions. The electron density differences $\Delta \rho_{\mathbf{a}}$ and $\Delta \rho_{\mathbf{b}}$ were defined by Eq. 2 and 3 , respectively. The absolute value of the contours is $0.01 e \AA^{-3}$, where blue is electron accumulation (area of negative differential charge) and red electron depletion (area of positive differential charge).

$\rho_{\text {mol2 }}$ are the electron densities of the two molecules isolated (without slab), respectively. The electron density of the slab and the molecular systems were optimized, while keeping their respective geometries fixed. Within these definitions, $\Delta \rho_{\mathbf{a}}$ gives the electron charge redistribution due to only the molecule-surface interactions, while $\Delta \rho_{\mathbf{b}}$ shows the electron charge difference induced by the molecule-surface as well as the molecule-molecule interactions.

From the visualization of $\Delta \rho_{\mathbf{a}}$ (Figure 4a) we conclude that the electron transfer between the molecules and the surface is relatively small in $\mathbf{T S} \mathbf{S}_{\text {couple }}$. This means that in the coupling of two molecules, the role of the substate is to limit the mobility of the molecules to two dimensions, without contributing to the reaction electronically. It should, however, be noted that the interactions between molecule and substrate are substantial in the intermediate states, where C-Ag bonds are formed. Thus, an important role of the substrate, for the overall reaction, is to stabilize the intermediate state $\mathbf{I n t} \mathbf{S} \mathbf{1}_{\text {cis }}$ following the coupling of two TEB molecules.

The considerably larger charge redistribution of $\Delta \rho_{\mathbf{b}}$ compared to $\Delta \rho_{\mathbf{a}}$ (Figure 4) reveals that the molecule-molecule interactions have a much greater influence on the electronic structure of $\mathbf{T S}$ couple than the molecule-surface interactions do. In particular, there is an area of electron accumulation connecting the two $\mathrm{C}$ atoms that are in the process of coupling. This negatively charged area is surrounded by a torus of electron depletion. We interpret this as a signature of the covalent bond being formed between the two molecules. A similar 


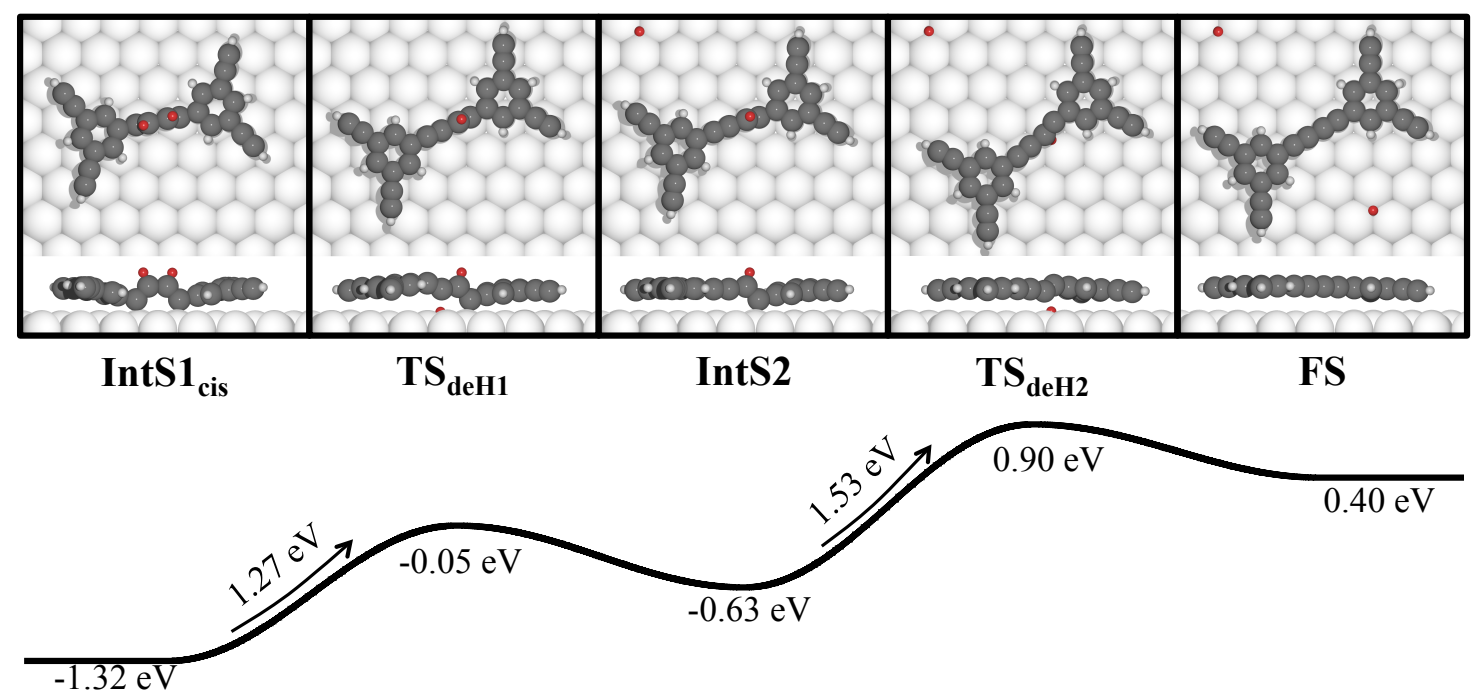

Figure 5: Reaction pathway for splitting-off two H-atoms, starting from the cis intermediate state IntS1 $\mathbf{1}_{\text {cis. }}$. To arrive at IntS2 it is necessary to go through $\mathbf{I n t S} \mathbf{1}_{\text {trans }}$ (see Figure 2) before reaching the first dehydrogenation transition state $\mathbf{T} \mathbf{S}_{\mathbf{d e H} \mathbf{1}}$, but for simplicity, only the effective barrier of this two-step process is indicated. The two hydrogen atoms being split-off are rendered in red to ease comparison. Energies of local minima and saddle points are given with respect to the initial state IS in Figure 2.

electron charge difference to $\Delta \rho_{\mathbf{b}}$ is found when artificially removing the surface from the calculations (see SI). This gives further evidence that the characteristics of $\Delta \rho_{\mathbf{b}}$ originate mainly from the molecule--molecule interactions.

Figure 5 describes the mechanism for abstracting the two hydrogen atoms, separating the intermediate $\mathbf{I n t S} \mathbf{1}_{\mathbf{c i s}}$ from the final state $\mathbf{F S}$. In order to split-off the first hydrogen, it is necessary to initially go through $\mathbf{I n t} \mathbf{S} \mathbf{1}_{\text {trans }}$ followed by the first dehydrogenation transition state $\mathbf{T S}_{\mathbf{d e H} \mathbf{1}}$. Note that it is also possible to go directly from $\mathbf{I n t S} \mathbf{1}_{\text {trans }}$ to IntS2. However, the energy difference between $\mathbf{T S}_{\mathbf{i s o}}$ and $\mathbf{T} \mathbf{S}_{\mathbf{d e H} \mathbf{1}}$ is $0.20 \mathrm{eV}$, which gives the Boltzmann factor of $\sim 10^{-3}$ at $330 \mathrm{~K}$. Thus, the $\mathbf{I n t} \mathbf{S} \mathbf{1}_{\text {trans }} \rightarrow \mathbf{I n t S} \mathbf{1}_{\text {cis }}$ transition is about 1000 times more likely than the $\mathbf{I n t S} \mathbf{1}_{\text {trans }} \rightarrow$ IntS2 transition, i.e., the majority of reactions proceed via $\mathbf{I n t S} \mathbf{1}_{\text {cis }}$. For simplicity, only the effective barrier for the overall IntS1 $\mathbf{1}_{\text {cis }} \rightarrow \mathbf{I n t S 2}$ process is indicated, defined as the energy difference between $\mathbf{T} \mathbf{S}_{\mathbf{d e H 1}}$ and $\mathbf{I n t} \mathbf{S} \mathbf{1}_{\mathbf{c i s}}$. The intermediate state IntS2 is chosen with the dissociated hydrogen away from the molecule. Thus, there are a few diffusion steps of the $\mathrm{H}$ atom over the $\mathrm{Ag}(111)$ surface which have been left out 
from the pathway in Figure 5, and the same holds for the final step from IntS2 to FS. To abstract the second hydrogen atom, the remaining $\mathrm{C}-\mathrm{Ag}$ bond has to be broken, enabling the rotation of the $\mathrm{CH}$ group such that the $\mathrm{H}$-atom is pointing towards the surface, which is then split-off. The second dehydrogenation $(\mathbf{I n t S 2} \rightarrow \mathbf{F S})$ is a single-barrier process, as no stable intermediate exists having the C-Ag bond broken, but with the hydrogen still bonded to the molecule.

The dehydrogenation steps imply appreciable barriers: $1.27 \mathrm{eV}$ and $1.53 \mathrm{eV}$ to split off the first and second hydrogen atom, respectively. The relatively large barriers originate from the fact that in both processes, one needs to break not only the $\mathrm{C}-\mathrm{H}$ bond, but also a chemical bond between the molecule and the surface. Furthermore, the dehydrogenation steps are endothermic. From the simple argument that the system wants to minimize its energy, it would be expected that in thermal equilibrium most of the dimerized molecules occur in IntS1 $_{\text {cis }}$ rather than FS. However, by comparing STM simulations of the intermediates to the final state of the reaction, it becomes clear that only FS reproduces the experimental high-resolution image, see Figure 6 . The existance of neither $\mathbf{I n t S} \mathbf{1}_{\text {cis }}$ nor IntS2 in the STM data is consistent with the assumption that the dehydrogenation steps are irreversible, meaning that the hydrogen atoms on the surface do not recombine with the dehydrogenated molecules. Considering the high diffusivity of $\mathrm{H}$ atom on $\operatorname{Ag}(111)$, it is suggested that the formation and subsequent desorption of molecular hydrogen, known to occur at temperatures below $200 \mathrm{~K},{ }^{33}$ is kinetically favored.

We have concluded that the TEB dimerization gives the final product FS in Figure 5. However, in particular the second dehydrogenation barrier, is too large considering that the reaction occurs in experiments at temperatures of $330 \mathrm{~K}$. However, the barrier of $1.53 \mathrm{eV}$ includes only the $0 \mathrm{~K}$ potential energy surface. If instead calculating free energies, using the quasi-harmonic approximation, ${ }^{50}$ the barrier is reduced to $1.36 \mathrm{eV}$ at $330 \mathrm{~K}$. In other words, the vibrational enthalpy and entropy enhance the probability of the transition from IntS2 to $\mathbf{F S}$, and the same is expected to be the case for the $\mathbf{I n t S} \mathbf{1}_{\mathbf{c i s}} \rightarrow \mathbf{I n t S 2}$ transition. 

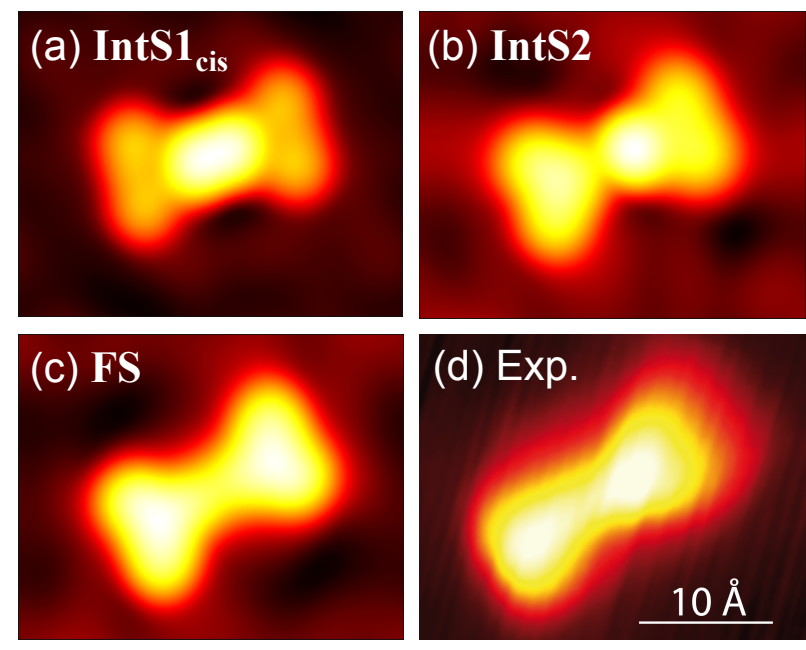

Figure 6: Simulated STM images (a-c) of the two intermediate states along the reaction path in Figure 5, as indicated, compared to a high-resolution experimental STM image (d) of a TEB dimer. The simulated images were obtained for $\mathrm{U}=-0.6 \mathrm{~V}$, and the experimental scanning parameters were $\mathrm{U}=-0.3 \mathrm{~V}, \mathrm{I}=0.1 \mathrm{nA}$. The images are of the size $2.9 \times 2.3 \mathrm{~nm}^{2}$.

Another aspect we need to take into account is how well the adsorbed molecules are described in the calculations. The barrier heights for the dehydrogenation steps are highly dependent on how strongly the molecule chemisorbs to the surface in the intermediate states: the stronger $\mathrm{C}-\mathrm{Ag}$ bond is, the higher is the dehydrogenation barrier. The van der Waals density functional with optB86b exchange (optB86b/vdWDF) used in this work has shown to accurately describe distances in organometallic contacts, ${ }^{51}$ as well as the adsorption height of 3,4,9,10-perylene-tetracarboxylic acid (PTCDA) on $\mathrm{Ag}(111)$ (see Supporting Information). At the same time, this functional produces a too deep interaction potential ${ }^{51}$ resulting in the overestimation of the binding energy. Consistently, in our recent work we find that the adsorption energy of a single TEB molecule on $\mathrm{Ag}(111)$ is larger than indicated by experiments. ${ }^{49}$ The too deep interaction potential could have two origins, namely an overestimate in non-local correlation (vdW interaction) and a too attractive exchange. In any case, both deficiencies would enhance the computed strength of a chemical bond to the surface. To test the hypothesis of the over-bonded chemisorbed intermediates in Figure 5, we performed calculations without vdW interactions, with the commonly used PBE functional. ${ }^{52}$ Interestingly, the PBE functional lowers the barrier by $0.15 \mathrm{eV}$, showing that reducing the interaction 
between molecule and surface indeed affects the barrier favorably.

The barrier heights of the IntS2 $\rightarrow$ FS transition, as calculated with the different methods, are summarized in Table 1. For an estimate of the real rate-limiting barrier, we suggest that the overestimation of molecule-surface interactions and the vibrational contribution to the free energy act additively to lowering the barrier of the dehydrogenation steps. We hypothesize that the true value might be near to $1.2-1.3 \mathrm{eV}$.

Table 1: The barrier height of the second dehydrogenation reaction (Figure 5) as calculated with optB86b/vdWDF functional, the optB86b/vdWDF functional including vibrational enthalpy and entropy at $330 \mathrm{~K}$, as well as the PBE functional.

\begin{tabular}{lc}
\hline & $E\left(\mathbf{T S}_{\mathbf{d e H 2}}\right)-E($ IntS2 $)$ \\
\hline \hline optB86b/vdWDF & $1.53 \mathrm{eV}$ \\
optB86b/vdWDF + vib. (330 K) & $1.36 \mathrm{eV}$ \\
PBE & $1.38 \mathrm{eV}$ \\
\hline
\end{tabular}

Several alternative mechanisms could, in principle, be operational during the final, critical steps of the homo-coupling process. For example, in the case of the dehydrogenation of the TEB monomer in Figure 1 it was found that a hydrogen adatom significantly lowers the barrier. However, in the case of the dehydrogenation of the IntS2 state, the hydrogen assisted reaction gives a barrier of $1.61 \mathrm{eV}$ (see SI), thus less favorable than the corresponding reaction on the atomically flat surface in Figure 5. We also considered a further tautomerization reaction as well as an Ag adatom catalyzed process, which are described in detail in the SI. However, both of these alternatives are connected with considerably larger barriers than the dehydrogenation path in Figure 5, supporting that the formation of the TEB dimer on $\operatorname{Ag}(111)$ follows the reaction path presented here. Furthermore, we expect that this is the generic mechanism for the homo-coupling of terminal alkynes on close-packed noble metal surfaces.

The pathway elucidated here is also helpful for the interpretation of our recent results on coupling reactions starting with the pre-synthesized TEB dimer species. ${ }^{20}$ We found additional reactions of terminal alkyne groups connecting to the butadiyne bridges of the 
original dimer (see Supporting Information Scheme S1). Such reactions could be explained by first going back from FS to IntS2 where the $\mathrm{H}$ atom necessary for the reversibility is provided by the attacking third alkyne. Then tautomerization steps could bring the $\mathrm{H}$ atom to the positions necessary to explain the different final products, featuring some $\mathrm{sp}_{2}$ carbons.

We would like to emphasize that the mechanism may be completely different on other, more reactive surfaces. The plethora of products recently found on $\mathrm{Cu}(111),{ }^{19}$ thus for conditions nearer to those of the Glaser-Hay reaction in wet chemistry, indicates marked differences to the present situation. Under the conditions applicable here, in our experiments combining STM and X-ray photoelectron spectroscopy (XPS) ${ }^{17}$ we found no indications

for Ag-acetylide intermediates, neither s nor pi-bonded modes ${ }^{28-30}$ playing a role for the butadiyne formation. This contrasts the pathway of the surface-supported Ullmann coupling reaction, where such organometallic species were directly observed with STM. ${ }^{15,53}$

\section{Conclusion}

In conclusion, we have unraveled the mechanisms behind the homo-coupling reaction between terminal alkynes on the $\operatorname{Ag}(111)$ surface. The role of the substrate is both to constrain the molecular motion in two dimensions, as well as to stabilize the coupled intermediate state prior to the dehydrogenation. However, no significant chemical contribution, in terms of electron transfer, in the actual C-C bond formation was revealed. The insights provided here contribute significantly to the knowledge necessary for the rational design and construction of low-dimensional hydrocarbon material with atomic precision by surface-confined reactions.

\section{Acknowledgement}

Funding was provided by the Swedish Research Council and the ERC Advanced Grant MolArt (No. 247299). Computer recourses were allocated by the National Supercomputer Centre, Sweden through SNAC and the MATTER consortium. 


\section{Supporting Information Available}

Additional figures, schemes and computational details are provided in the supporting information. This material is available free of charge via the Internet at http://pubs.acs . org/.

\section{References}

(1) Sakamoto, J.; van Heijst, J.; Lukin, O.; Schlüter, A. D. Two-Dimensional Polymers: Just a Dream of Synthetic Chemists? Angew. Chem. Int. Ed. 2009, 48, 1030-1069.

(2) Franc, G.; Gourdon, A. Covalent Networks Through On-Surface Chemistry in UltraHigh Cacuum: State-of-the-Art and Recent Developments. Phys. Chem. Chem. Phys. 2011, 13, 14283-14292.

(3) Colson, J. W.; Dichtel, W. R. Rationally Synthesized Two-Dimensional Polymers. Nature Chem. 2013, 5, 453-465.

(4) Lafferentz, L.; Ample, F.; Yu, H.; Hecht, S.; Joachim, C.; Grill, L. Conductance of a Single Conjugated Polymer as a Continuous Function of Its Length. Science 2009, 323, $1193-1198$.

(5) Bieri, M.; Treier, M.; Cai, J.; Aït-Mansour, K.; Ruffieux, P.; Gröning, O.; Gröning, P.; Kastler, M.; Rieger, R.; Feng, X. et al. Porous Graphenes: Two-Dimensional Polymer Synthesis with Atomic Precision. Chem. Commun. 2009, 6919-6921.

(6) Cai, J.; Ruffieux, P.; Jaafar, R.; Bieri, M.; Braun, T.; Blankenburg, S.; Muoth, M.; Seitsonen, A. P.; Saleh, M.; Feng, X. et al. Atomically Precise Bottom-Up Fabrication of Graphene Nanoribbons. Nature 2010, 466, 470-473.

(7) Bieri, M.; Blankenburg, S.; Kivala, M.; Pignedoli, C. a.; Ruffieux, P.; Müllen, K.; Fasel, R. Surface-Supported 2D Heterotriangulene Polymers. Chem. Commun. 2011, 47, 10239-10241. 
(8) Treier, M.; Pignedoli, C. A.; Laino, T.; Rieger, R.; Müllen, K.; Passerone, D.; Fasel, R. Surface-Assisted Cyclodehydrogenation Provides a Synthetic Route Towards Easily Processable and Chemically Tailored Nanographenes. Nature Chem. 2011, 3, 61-67.

(9) Fan, Q.; Wang, C.; Han, Y.; Zhu, J.; Hieringer, W.; Kuttner, J.; Hilt, G.; Gottfried, J. M. Surface-Assisted Organic Synthesis of Hyperbenzene Nanotroughs. Angew. Chem. Int. Ed. 2013, 52, 4668-4672.

(10) Sun, Q.; Zhang, C.; Li, Z.; Kong, H.; Tan, Q.; Hu, A.; Xu, W. On-Surface Formation of One-Dimensional Polyphenylene through Bergman Cyclization. J. Am. Chem. Soc. 2013, 135, 8448-8451.

(11) Novoselov, K. S.; Fal'ko, V. I.; Colombo, L.; Gellert, P. R.; Schwab, M. G.; Kim, K. A Roadmap for Graphene. Nature 2012, 490, 192-200.

(12) Diederich, F.; Kivala, M. All-Carbon Scaffolds by Rational Design. Adv. Mater. 2010, 22, 803-812.

(13) Grill, L.; Dyer, M.; Lafferentz, L.; Persson, M.; Peters, M. V.; Hecht, S. NanoArchitectures by Covalent Assembly of Molecular Building Blocks. Nature Nanotech. 2007, 2, 687-691.

(14) Björk, J.; Hanke, F.; Stafström, S. Mechanisms of Halogen-Based Covalent SelfAssembly on Metal Surfaces. J. Am. Chem. Soc. 2013, 135, 5768-5775.

(15) Wang, W.; Shi, X.; Wang, S.; Van Hove, M. a.; Lin, N. Single-Molecule Resolution of an Organometallic Intermediate in a Surface-Supported Ullmann Coupling Reaction. J. Am. Chem. Soc. 2011, 133, 13264-13267.

(16) Wang, S.; Wang, W.; Lin, N. Visualization and Manipulation of Individual Dopant States in Single Conjugated Oligomers. ACS Nano 2012, 6, 3404-3410. 
(17) Zhang, Y.-Q.; Kepčija, N.; Kleinschrodt, M.; Diller, K.; Fischer, S.; Papageorgiou, A. C.; Allegretti, F.; Björk, J.; Klyatskaya, S.; Klappenberger, F. et al. HomoCoupling of Terminal Alkynes on a Noble Metal Surface. Nat. Commun. 2012, 3, 1286.

(18) Gao, H.-Y.; Wagner, H.; Zhong, D.; Franke, J.-H.; Studer, A.; Fuchs, H. Glaser Coupling at Metal Surfaces. Angew. Chem. Int. Ed. 2013, 52, 4024-4028.

(19) Eichhorn, J.; Heckl, W. M.; Lackinger, M. On-Surface Polymerization of 1,4diethynylbenzene on $\mathrm{Cu}(111)$. Chem. Commun. 2013, 49, 2900-2902.

(20) Cirera, B.; Zhang, Y.-Q.; Klyatskaya, S.; Ruben, M.; Klappenberger, F.; Barth, J. V. Surface-Assisted Two Dimensional Self-Assembly and Catalytic Homo-Coupling of the Terminal Alkyne 1,4-Bis(3,5-bisethynylphenyl)butadiyne-1,3 on $\mathrm{Ag}(111)$. ChemCatChem 2013, 5, 3281-3288.

(21) Matena, M.; Stöhr, M.; Riehm, T.; Björk, J.; Martens, S.; Dyer, M. S.; Persson, M.; Lobo-Checa, J.; Müller, K.; Enache, M. et al. Aggregation and Contingent Metal/Surface Reactivity of 1,3,8,10-Tetraazaperopyrene (TAPP) on $\mathrm{Cu}(111)$. Chem. Eur. J. 2010, 16, 2079-2091.

(22) Hanke, F.; Haq, S.; Raval, R.; Persson, M. Heat to Connect: Surface Commensurability Directs Organometallic One-Dimensional Self-Assembly. ACS Nano 2011, 5, 90939103.

(23) Nguyen, M.-T.; Pignedoli, C. A.; Passerone, D. An Ab Initio Insight into the Cu(111)Mediated Ullmann Reaction. Phys. Chem. Chem. Phys. 2011, 13, 154-160.

(24) Blankenburg, S.; Cai, J.; Ruffieux, P.; Jaafar, R.; Passerone, D.; Feng, X.; Fasel, R.; Pignedoli, C. A. Intraribbon Heterojunction Formation in Ultranarrow Graphene Nanoribbons. ACS Nano 2012, 2020-2025. 
(25) Björk, J.; Hanke, F. Towards Design Rules for Covalent Nanostructures on Metal Surfaces. Chem. Eur. J. 2014, 20, 928-934.

(26) Glaser, C. Beiträge zur Kenntnis des Acetenylbenzols. Ber. Dtsch. Chem. Ges. 1869, 2, 422-424.

(27) Hay, A. S. Oxidative Coupling of Acetylenes .2. J. Org. Chem. 1962, 27, 3320-3321.

(28) Halbes-Letinois, U.; Weibel, J.-M.; Pale, P. The Organic Chemistry of Silver Acetylides. Chem. Soc. Rev. 2007, 36, 759-769.

(29) Long, N. J.; Williams, C. K. Metal Alkynyl Sigma Complexes: Synthesis and Materials. Angew. Chem. Int. Ed. 2003, 42, 2586-2617.

(30) Yamamoto, Y. Silver-Catalyzed C(sp)-H and C(sp)-Si Bond Transformations and Related Processes. Chem. Rev. 2008, 108, 3199-3222.

(31) Siemsen, P.; Livingston, R.; Diederich, F. Acetylenic Coupling: A Powerful Tool in Molecular Construction. Angew. Chem. Int. Ed. 2000, 39, 2632-2657.

(32) Montoya, A.; Schlunke, A.; Haynes, B. S. Reaction of Hydrogen With Ag(111): Binding States, Minimum Energy Paths, and Kinetics. J. Phys. Chem. B 2006, 110, 1714517154 .

(33) Zhou, X.; White, J.; Koel, B. Chemisorption of Atomic Hydrogen on Clean and Clcovered $\mathrm{Ag}(111)$. Surf. Sci. 1989, 218, 201-210.

(34) Gao, H.-Y.; Franke, J.-H.; Wagner, H.; Zhong, D.; Held, P.-a.; Studer, A.; Fuchs, H. Effect of Metal Surfaces in On-Surface Glaser Coupling. J. Phys. Chem. C 2013, 117, $18595-18602$.

(35) Fomina, L.; Vazquez, B.; Tkatchouk, E.; Fomine, S. The Glaser Reaction Mechanism. A DFT Study. Tetrahedron 2002, 58, 6741-6747. 
(36) Vilhelmsen, M. H.; Jensen, J.; Tortzen, C. G.; Nielsen, M. B. The Glaser-Hay Reaction: Optimization and Scope Based on 13 C NMR Kinetics Experiments. Eur. J. Org. Chem. 2013, 701-711.

(37) van Gelderen, L.; Rothenberg, G.; Roberto Calderone, V.; Wilson, K.; Raveendran Shiju, N. Efficient Alkyne Homocoupling Catalysed by Copper Immobilized on Functionalized Silica. Appl. Organometal. Chem. 2013, 27, 23-27.

(38) Kresse, G.; Furthmüller, J. Efficient Iterative Schemes for Ab Initio Total-Energy Calculations Using a Plane-Wave Basis Set. Phys. Rev. B 1996, 54, 11169-11186.

(39) Blöchl, P. E. Projector Augmented-Wave Method. Phys. Rev. B 1994, 50, 17953-17979.

(40) Kresse, G.; Joubert, D. From Ultrasoft Pseudopotentials to the Projector AugmentedWave Method. Phys. Rev. B 1999, 59, 1758-1775.

(41) Dion, M.; Rydberg, H.; Schröder, E.; Langreth, D. C.; Lundqvist, B. I. Van der Waals Density Functional for General Geometries. Phys. Rev. Lett. 2004, 92, 246401-246404.

(42) Klimeš, J.; Bowler, D.; Michaelides, A. Van der Waals density Functionals Applied to Solids. Phys. Rev. B 2011, 83, 195131-195143.

(43) Henkelman, G.; Uberuaga, B. P.; Jónsson, H. A Climbing Image Nudged Elastic Band Method for Finding Saddle Points and Minimum Energy Paths. J. Chem. Phys. 2000, 113, 9901-9904.

(44) Henkelman, G.; Jónsson, H. Improved Tangent Estimate in the Nudged Elastic Band Method for Finding Minimum Energy Paths and Saddle Points. J. Chem. Phys. 2000, 113, 9978-9985.

(45) Henkelman, G.; Jónsson, H. A Dimer Method for Finding Saddle Points on High Dimensional Potential Surfaces Using Only First Derivatives. J. Chem. Phys. 1999, 111, 7010-7022. 
(46) Kästner, J.; Sherwood, P. Superlinearly Converging Dimer Method for Transition State Search. J. Chem. Phys. 2008, 128, 014106-014111.

(47) Tersoff, J.; Hamann, D. R. Theory and Application for the Scanning Tunneling Microscope. Phys. Rev. Lett. 1983, 50, 1998-2001.

(48) Lorente, N.; Persson, M. Theoretical Aspects of Tunneling-Current-Induced Bond Excitation and Breaking at Surfaces. Faraday Discuss. 2000, 277-290.

(49) Kepčija, N.; Zhang, Y.-Q.; Kleinschrodt, M.; Björk, J.; Klyatskaya, S.; Klappenberger, F.; Ruben, M.; Barth, J. V. Steering On-Surface Self-Assembly of High-Quality Hydrocarbon Networks with Terminal Alkynes. J. Phys. Chem. C 2013, 117, 39873995.

(50) Jensen, F. Introduction to Computational Chemistry; John Wiley \& Sons, Ltd, 1999.

(51) Mittendorfer, F.; Garhofer, A.; Redinger, J.; Klimeš, J.; Harl, J.; Kresse, G. Graphene on $\mathrm{Ni}(111)$ : Strong Interaction and Weak Adsorption. Phys. Rev. B 2011, 84, 201401201404.

(52) Perdew, J.; Burke, K.; Ernzerhof, M. Generalized Gradient Approximation Made Simple. Phys. Rev. Lett. 1996, 77, 3865-3868.

(53) Di Giovannantonio, M.; El Garah, M.; Lipton-Duffin, J.; Meunier, V.; Cardenas, L.; Fagot Revurat, Y.; Cossaro, A.; Verdini, A.; Perepichka, D. F.; Rosei, F. et al. Insight into Organometallic Intermediate and Its Evolution to Covalent Bonding in SurfaceConfined Ullmann Polymerization. ACS Nano 2013, 7, 8190-8198. 